\title{
Lichen sclerosus of the glans penis
}

\section{Katarzyna Borowska', Piotr Brzeziński²}

${ }^{1}$ Department of Histology and Embryology with Experimental Cytology Unit, Medical University of Lublin, Lublin, Poland, ${ }^{2}$ Department of Physiotherapy and Medical Emergency, Faculty of Health Sciences, Pomeranian Academy, Slupsk, Poland

Corresponding author: Piotr Brzeziński, MD PhD, E-mail: brzezoo77@yahoo.com

\begin{abstract}
Lichen sclerosus (LS) is a chronic inflammatory skin disease of genital and extragenital skin. LS is associated with a potential for atrophy, destructive scarring, functional impairment, and malignant evolution. Spontaneous remissions are rare. Therefore, early diagnosis, prompt treatment, and long-term follow-up of affected patients are mandatory. We present a case of 53-year-old man with lichen sclerosus of the glans penis.
\end{abstract}

Key words: Lichen sclerosus, Male, Genital

\section{INTRODUCTION}

Lichen sclerosus (LS) is a chronic, inflammatory, mucocutaneous disorder of genital and extragenital skin. LS is associated with a potential for atrophy, destructive scarring, functional impairment, and malignant evolution (penile intraepithelial neoplasia) $[1,2]$. In the early stages of LS the histologic picture may be uncharacteristic. The classic histologic features of more advanced uncomplicated LS are hyperkeratosis, epidermal atrophy with flattening of the rete ridges, vacuolar interface changes, loss of elastic fibers, and hyalinization of the lamina propria with an underlying lymphocytic infiltrate [3,4]. Therefore, early diagnosis, prompt treatment, and long-term follow-up of affected patients are mandatory. Spontaneous remissions are rare.

\section{CASE REPORT}

A 53-year-old man presented to the dermatology department with a skin lesions on the glans penis (Fig. 1). He had been receiving dermatologic treatment of lichen planus on the glans penis for 8 years.

At the initial stage, was observe on the foreskin porcelain hypochrome plates, with impairment glans, balano-preputial groove, frenulum and meatus.

The patient is giving dysuria and cracks due to epidermal fragility.

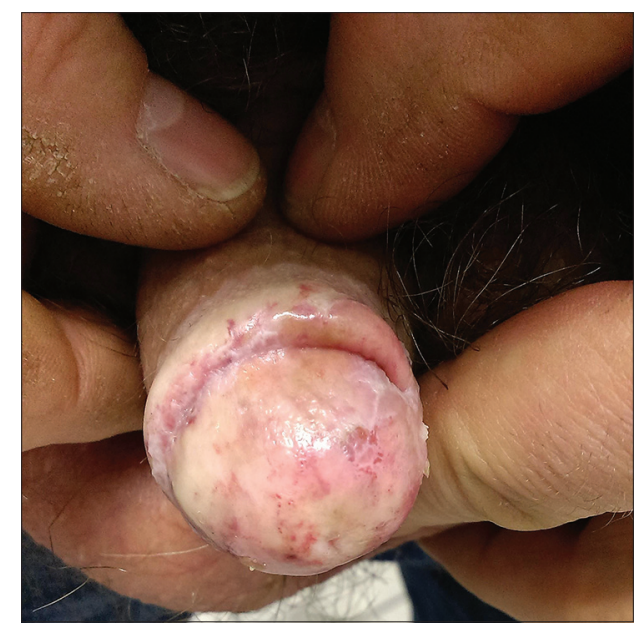

Figure 1: Lichen sclerosus of the glans penis in 53-year-old man.

Treated for an enlarged prostate gland. In laboratory tests (including morphology, anti-HCV, anti-HBS), no abnormalities were observed.

\section{DISCUSSION}

Lichen sclerosus (LS), previously known as balanitis xerotica obliterans, most often occurs in the 4th and 5 th decade of life, but has also been described in adolescents and in extremes of age [5]. In stuby of Nyati et al in patiients non-venereal genital dermatoses in one clinical center was found LS in $2 \%$ cases [6]. The exact etiology of LS remains unclear; theories

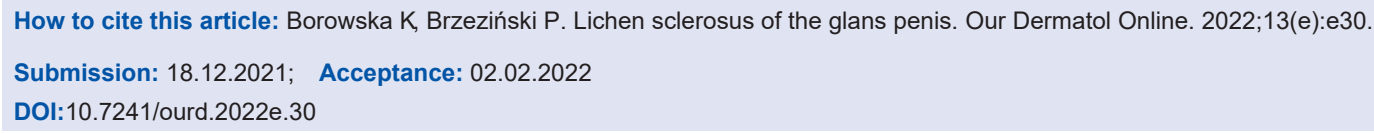


have hypothesized a possible autoimmune element. The role of the foreskin is unknown, but it most likely contributes to the development of disease through the accumulation of epithelial debris and secretions in the fold between the foreskin and penis proximal to the coronal sulcus. This accumulated material then causes chronic physical irritation, chronic balanitis or subclinical trauma with subsequent Koebner phenomenon, where skin lesions appear along the lines of trauma [5,7]. The commonest symptoms associated with penile lichen sclerosus is a color change to a shade of red or shimmering white of the glans penis, foreskin (prepuce) and coronal sulcus with thickening (induration) of the glans and foreskin. These symptoms are not specific [5,6]. Lichen sclerosus most commonly affects the glans penis and prepuce, such that a sclerotic white ring at the tip of the prepuce is diagnostic. The frenulum, urethral meatus, fossa navicularis (most distal dilated portion of the urethra) and penile shaft may become involved [5]. With further disease progression, the glans penis may become adherent to the prepuce with fi brous replacement of the coronal sulcus and frenulum, leading to phimosis [5-7]. The concept of LS as a premalignant lesion remains contentious. Lichen sclerosus can be managed by both conservative and surgical techniques [5].

\section{Consent}

The examination of the patient was conducted according to the principles of the Declaration of Helsinki.
The authors certify that they have obtained all appropriate patient consent forms, in which the patients gave their consent for images and other clinical information to be included in the journal. The patients understand that their names and initials will not be published and due effort will be made to conceal their identity, but that anonymity cannot be guaranteed.

\section{REFERENCES}

1. Garaffa G, Shabbir M, Christopher N, Minhas S, Ralph DJ. The surgical management of lichen sclerosus of the glans penis: our experience and review of the literature. J Sex Med. 2011;8:1246-53.

2. Puri N, Puri A. A study on non venereal genital dermatoses in north India. Our Dermatol Online. 2013;4:304-7.

3. Nyati A, Singh A, Mohta A, Kushwaha RK, Gupta D, Jain SK. A study of the pattern of non-venereal genital dermatoses in male patients at a Tertiary Care Centre from Hadoti region of Rajasthan. Our Dermatol Online. 2021;12:e87.

4. Murphy R. Lichen sclerosus. Dermatol Clin. 2010;28:707-15.

5. Funaro D. Lichen sclerosus: a review and practical approach. Dermatol Ther. 2004;17:28-37.

6. Regauer S, Liegl B, Reich O. Early vulvar lichen sclerosus: a histopathological challenge. Histopathology. 2005;47:340-7.

7. Regauer S, Liegl B, Reich O, Pickel H, Beham-Schmid C. Lichen sclerosus vulvae. Die besondere Bedeutung der klinischen und histopathologischen Früherkennung [Vulvar lichen sclerosus. The importance of early clinical and histological diagnosis]. Hautarzt. 2004;55:158-64.

Copyright by Katarzyna Borowska, et al. This is an open-access article distributed under the terms of the Creative Commons Attribution License, which permits unrestricted use, distribution, and reproduction in any medium, provided the original author and source are credited.

Source of Support: Nil, Conflict of Interest: None declared. 